\title{
Korrelation erhöhter longitudinaler und transversaler Dehnungsempfindlichkeit granularer Metalle am Beispiel von Platin-Aluminiumoxid-Dünnschichten
}

\author{
S. Schwebke, G. Schultes \\ HTW Saar, Hochschule für Technik und Wirtschaft des Saarlandes, \\ Goebenstr. 40, 66117 Saarbrücken \\ silvan.schwebke@htwsaar.de
}

\section{Zusammenfassung}

Die Dehnungsempfindlichkeit cogesputterter Cermet-Dünnschichten aus Platin und Aluminiumoxid $\left(\mathrm{Pt}: \mathrm{Al}_{2} \mathrm{O}_{3}\right)$ wird untersucht. Sie weisen gegenüber metallischen Schichten eine erhöhte Sensitivität $(k$ bis zu 10) auf, zeigen jedoch nicht nur bei longitudinaler Dehnung einen k-Faktor $k_{L}$, sondern bei Dehnung orthogonal zur Stromflussrichtung einen transversalen k-Faktor $k_{T}$. Die Querempfindlichkeit beträgt $k_{T} / k_{L} \approx 0,6 \ldots 0,7$.

Schichtanalysen mittels EDX, XRD und TEM zeigen einen Metallanteil um 25 Atomprozent und eine kristalline Platinphase in Form von Partikeln der Größe $5 \mathrm{~nm}$, umgeben von einer amorphen $\mathrm{Al}_{2} \mathrm{O}_{3}-\mathrm{Matrix}$. Diese Struktur wird in einem Modell abgebildet, das in Zusammenhang mit einem Widerstandsgesetz die numerische Berechnung von $k_{L}$ und $k_{T}$ ermöglicht. Das Modell liefert einen Ansatz, die transversale Empfindlichkeit zu erklären: Sie ergibt sich zum einen aus der granularen Struktur, bei der Elektronentransport von Metallpartikel zu Metallpartikel stattfindet, zum anderen aus der Unordnung der Partikel, die Umwege in den Pfaden der Elektronenleitung begünstigt. Der Mechanismus, der den erhöhten kFaktor $k_{L}$ ermöglicht, bedingt damit auch die Querempfindlichkeit.

Verglichen mit klassischen metallischen Dünnschichten sorgt die Querempfindlichkeit der Schicht für neue Randbedingungen des Einsatzes; sie bietet jedoch die Aussicht auf Verbesserungen, zum Beispiel bei Drucksensormembranen, bei denen sie die Sensitivität zusätzlich erhöhen kann.

Keywords: Dünnschichttechnik, Dehnungssensorik, piezoresistive Materialien, Cermets, granulare Metalle

\section{Einleitung}

Zur Messung von Dehnungen in Kraft- oder Drucksensoren sind metallische Dünnschichten, insbesondere solche aus $\mathrm{NiCr}$, seit vielen Jahrzehnten Stand der Technik [1] [2]. Sie weisen eine geometrisch bedingte Dehnungsempfindlichkeit (k-Faktor) von etwa 2 auf. Auf der Suche nach Dünnschichten mit erhöhter Dehnungsempfindlichkeit spielt die Materialklasse der granularen metallhaltigen Schichten in der Forschung eine wichtige Rolle. Darunter fallen Metall-Kohlenstoffschichten [3] [4] (MetallDLC, Metall mit amorphem Kohlenstoff), MetallKeramik-Schichten (Cermets) und andere Kompositmaterialen, in denen Metalle mit nichtmetallischen Stoffen, beispielsweise Metalloxiden und -nitriden, kombiniert werden.

Für gewöhnlich wird die Dehnungsempfindlichkeit ermittelt, indem eine Widerstandsstruktur mit einer Dehnung parallel zur Stromrichtung beauf- schlagt wird; dies liefert den k-Faktor

$$
k=\frac{1}{\varepsilon} \frac{\Delta R}{R},
$$

mit dem Widerstand $R$, dessen Änderung $\Delta R$ und der Dehnung $\varepsilon=\Delta L / L$. Erfolgt die Dehnung einer Widerstandsstruktur in Richtung der Stromflussrichtung, ist der longitudinale k-Faktor $k_{L}$ wirksam.

Einige Untersuchungen haben zusätzlich die transversale Dehnungsempfindlichkeit $k_{T}$ zum Gegenstand: Hier wird die Dehnung orthogonal zum Stromfluss aufgeprägt. Klassische dehnungsempfindliche Metallschichten aus $\mathrm{Ni}$ $\mathrm{Cr}\left(k_{L} \approx 2\right)$ weisen eine vernachlässigbar geringe transversale Empfindlichkeit auf $\left(k_{T} / k_{L}=\right.$ $0)$. Dagegen zeigen experimentelle Ergebnisse für hochempfindliche Schichten eine deutliche transversale Dehnungsempfindlichkeit [5]. Untersuchungen verschiedener Schichten liefern abhängig von ihrer Struktur, insbesondere der Unordnung im granularen Aufbau, Werte von $k_{T} / k_{L}=0,5$ für Nickel-Kohlenstoff [6] bis 
hin zu $k_{T} / k_{L}=1$ bei gesinterten DickschichtWiderständen [7].

Für alle genannten Schichten gilt, dass mit erhöhter longitudinaler Dehnungsempfindlichkeit auch eine transversale Empfindlichkeit einhergeht. Zugleich zeichnet sich die Struktur der Schichten durch einen granularen Aufbau aus, bei dem ungeordnet vorliegende metallische Partikel durch Trennwände aus anderen, schlechter leitfähigen Materialien (z.B. Kohlenstofflagen oder Oxiden) separiert sind.

Erklärungen für die Eigenschaften solcher Schichten berücksichtigen den granularen Aufbau mit seiner geometrischen Unordnung. Grimaldi et al. [8] analysierten ein zufälliges Bond-Perkolations-Netzwerk als Modell für die transversale Empfindlichkeit in DickschichtWiderständen; dabei ergab sich $k_{T} / k_{L}=1$. Jiang et al. [9] lösen für ein Gold-NanopartikelSystem ein Netzwerk aus zufällig verteilten Widerständen mittels kirchhoffscher Gleichungen, um das Verhältnis $k_{T} / k_{L}$ zu ermitteln, das von Null mit steigender Unordnung bis zu einem Wert von 1 wächst.

Schichten mit transversaler Empfindlichkeit sind für die Ermittlung unbekannter zweiachsiger Spannungsfelder unvorteilhaft, da sich die Spannungen notwendigerweise in der Widerstandsänderung überlagern; eine Unterscheidung erfordert hier mindestens den Einsatz einer zweiten Sensorstruktur. Einen Vorteil stellen die Schichten jedoch dar in Messsituationen mit einachsigem Spannungsfeld, da ihre erhöhten Dehnungsempfindlichkeit für eine gegebene Dehnung ein gesteigertes Messsignal liefert oder es alternativ erlaubt, Bauteile starrer, d.h. auf eine geringere Dehnung auszulegen. Besonders vorteilhaft ist die transversale Empfindlichkeit der Schicht, wenn Messungen in einem zweiachsigem Spannungsfeld erfolgen, bei dem die zu ermittelnden Dehnungen eindeutig zusammenhängen und das gleiche Vorzeichen besitzen. Hier ergibt sich das Signal aus der Summe der longitudinalen und der transversalen Empfindlichkeit und erreicht damit eine nochmals erhöhte Sensitivität. Diese Situation ist etwa in den inneren Dehnungszonen von Drucksensor-Membranen gegeben.

Gegenstand unserer Untersuchung sind granulare Cermet-Dünnschichten aus Platin und Aluminiumoxid $\left(\mathrm{Pt}_{\mathrm{Al}} \mathrm{Al}_{2} \mathrm{O}_{3}\right)$. Diese werden durch RFCosputtern auf Glassubstraten abgeschieden. Die erzeugten Schichten werden hinsichtlich elektrischer und piezoresistiver Eigenschaften untersucht. Ihre Struktur wird durch Analyse mittels energiedispersiver Röntgenspektroskopie (EDX), Röntgenbeugung (XRD) und Transmissionselektronenmikroskopie (TEM) untersucht.
Tabelle 1: Parameter des RF-CosputterProzesses

\begin{tabular}{cccc}
\hline Prozess-Nr. & 1 & 2 & 3 \\
\hline Prozessdauer & $30 \mathrm{~min}$ & $30 \mathrm{~min}$ & $30 \mathrm{~min}$ \\
Prozessdruck & $0,6 \mathrm{~Pa}$ & $0,6 \mathrm{~Pa}$ & $0,6 \mathrm{~Pa}$ \\
Substrat-Bias & $-60 \mathrm{~V}$ & $-60 \mathrm{~V}$ & $-60 \mathrm{~V}$ \\
Leistung Pt & $60 \mathrm{~W}$ & $60 \mathrm{~W}$ & $70 \mathrm{~W}$ \\
Leistung $\mathrm{Al}_{2} \mathrm{O}_{3}$ & $240 \mathrm{~W}$ & $200 \mathrm{~W}$ & $200 \mathrm{~W}$ \\
Temperatur & $323^{\circ} \mathrm{C}$ & $316^{\circ} \mathrm{C}$ & $335^{\circ} \mathrm{C}$ \\
\hline
\end{tabular}

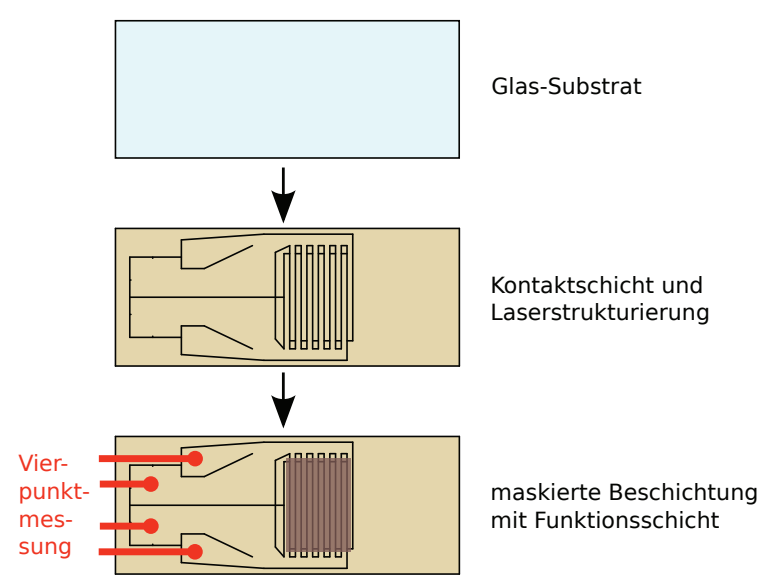

Abbildung 1: Eine Probe wird mit zuerst Kontaktschicht besputtert. Mit dem Pikosekundenlaser wird eine Interdigitalstruktur erzeugt. Mit einer Schattenmaske wird in einem definierten $\mathrm{Be}$ reich die Pt: $\mathrm{Al}_{2} \mathrm{O}_{3}$-Funktionsschicht aufgesputtert. Die elektrische Messung erfolgt über die vier markierten Kontakte.

Mit diesen Ergebnissen und Ansätzen aus der Literatur wird ein Modell der Schichtstruktur und der elektrisch-mechanischen Eigenschaften aufgestellt und numerisch in Monte-CarloSimulationen ausgewertet, um einen Erklärungsansatz für die Eigenschaften der Schicht zu finden.

\section{Experimente}

Die in Abb. 1 gezeigte Prozessabfolge umfasst die Sputterdeposition einer Kontaktschicht (CrNi$\mathrm{Ni}-\mathrm{Au}$ ) auf dem Borofloatsubstrat, die anschlieBend per Pikosekundenlaser strukturiert wird.

Prozessparameter des RF-Cosputter-Prozesses für die untersuchten $k_{L}-k_{T}$-Proben sind in Tab. 1 angegeben. Es wird von zwei separaten Targets (Durchmesser $100 \mathrm{~mm}$ ) gesputtert; die Substrate befanden sich auf einem geheizten rotierenden Teller in ca. $100 \mathrm{~mm}$ Abstand von den Targets.

Das verwendete Layout umfasst Kontaktstellen zur späteren elektrischen Messung und eine interdigitale Grabenstruktur, die im nächsten Schritt mit der Funktionsschicht gefüllt wird. Da- 


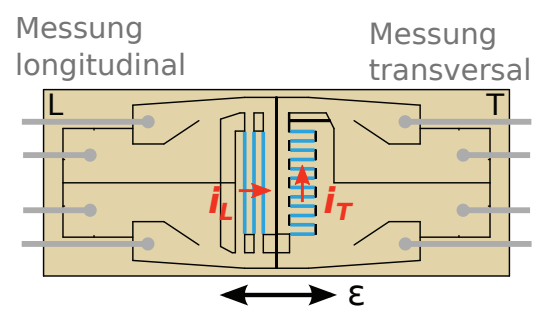

Abbildung 2: Doppeltes Interdigital-Layout mit longitudinaler (links) und transversaler (rechts) Dehnung der Funktionsschicht. Die wirksamen Funktionsschichtgräben sind blau hervorgehoben, die jeweilige Stromflussrichtung durch die Gräben ist durch rote Pfeile gekennzeichnet.

zu wird die Probe mit einer Schattenmaske, deren Öffnung den Bereich der Gräben freilässt, mit der $\mathrm{Pt}: \mathrm{Al}_{2} \mathrm{O}_{3}-\mathrm{Schicht}$ bedeckt. Aufgrund der Interdigitalstruktur fließt der Messstrom durch zahlreiche parallele Gräben; der Probenwiderstand wird damit auf etwa 1/4000 des sehr großen Flächenwiderstandes reduziert und erreicht Werte von einigen hundert Ohm.

Zur Charakterisierung werden die $30 \mathrm{~mm} \times$ $12 \mathrm{~mm} \times 0,5 \mathrm{~mm}$ großen Proben einseitig eingespannt und am freien Ende auf einen festen Radius von $1000 \mathrm{~mm}$ niedergedrückt, um eine Längsdehnung von $\varepsilon=0,25 \%$ zu erzeugen. Die entstehende Querdehnung liegt im Bereich weniger Prozent der Längsdehnung und ist damit vernachlässigbar gering. Die elektrische Messung erfolgt über die Vierleitermethode, um den Einfluss der Zuleitungswiderstände zu minimieren. Je nach Orientierung der Widerstandsgräben lassen sich Proben erzeugen, die bei gleichem Belastungsaufbau eine Messung der longitudinalen oder der transversalen Dehnungsempfindlichkeit erlauben. Für die zu vermessenden Proben wurde das in Abb. 2 dargestellte Layout entworfen. Es erlaubt je nach Einbau der Probe (Seite $L$ oder Seite $T$ mechanisch eingespannt und elektrisch kontaktiert) die Messung des longitudinalen oder des transversalen kFaktors. Hierbei ist die Richtung der Dehnung stets gleich, doch der Stromfluss durch die interdigitalen Gräben der Funktionsschicht ist einmal parallel, einmal orthogonal zur Dehnung.

Im Ergebnis zeigen die Pt: $\mathrm{Al}_{2} \mathrm{O}_{3}$-Schichten charakteristische Eigenschaften von Cermets: Sie sind hochohmig - bei einer Schichtdicke von $100 \mathrm{~nm}$ liegt der Flächenwiderstand mit Metallkonzentrationen um 25 Atomprozent bei etwa $3 \mathrm{M} \Omega$ und variiert mit dem Metallgehalt um einige Größenordnungen. Die Temperaturkoeffizienten des elektrischen Widerstandes (TKR) sind für alle Schichten mit erhöhten k-Faktoren stark negativ, bis hin zu etwa $-5 \cdot 10^{-3} \mathrm{~K}^{-1}$. Der longitudinale k-Faktor erreicht Werte bis etwa 10

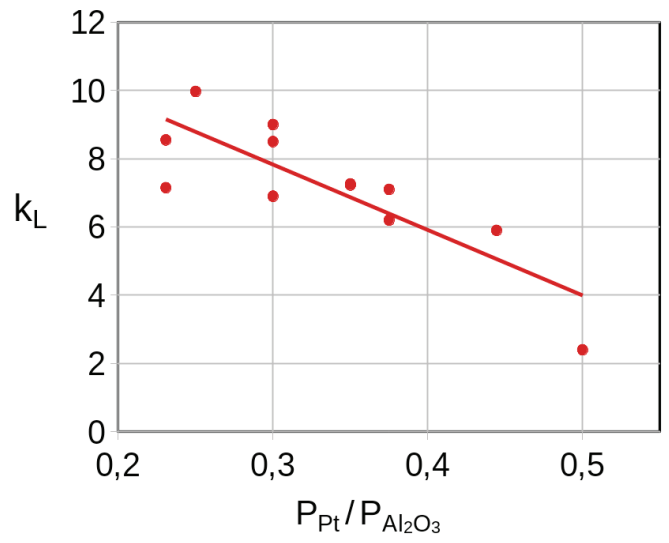

Abbildung 3: Longitudinaler k-Faktor für cogesputterte Pt: $\mathrm{Al}_{2} \mathrm{O}_{3}$-Schichten unterschiedlichen Metallgehalts (anhand des Verhältnisses der Sputterleistungen $P_{\mathrm{Pt}}$ und $P_{\mathrm{Al}_{2} \mathrm{O}_{3}}$ )

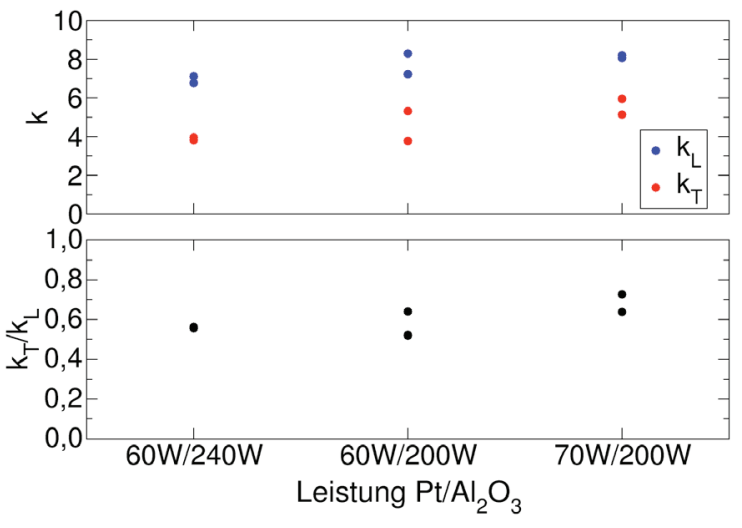

Abbildung 4: Experimentelle Ergebnisse der longitudinalen und transversalen k-Faktoren aus drei Prozessen mit unterschiedlichem Metallanteil. Oben: gemessene $k_{L}$ und $k_{T}$. Unten: Querempfindlichkeit als Verhältnis $k_{T} / k_{L}$.

und nimmt mit steigendem Metallgehalt ab, wie in Abb. 3 dargestellt. Abbildung 4 stellt Ergebnisse für das Verhältnis der transversalen zur longitudinalen Empfindlichkeit dar. Es liegt für die untersuchten Proben bei $k_{T} / k_{L} \approx 0,6 \ldots 0,7$. Die $\mathrm{Pt}: \mathrm{Al}_{2} \mathrm{O}_{3}$-Schicht liegt damit verglichen mit den eingangs beschriebenen Literaturergebnissen in einem mittleren Bereich der Querempfindlichkeit.

\section{Schichtstruktur}

In XRD-Analysen (siehe Abb. 5) zeigen die Schichten deutliche Peaks des Platin-Gitters, die jedoch gegenüber Literaturwerten leicht verschoben sind, was auf einen Einbau von Fremdatomen im Gitter hinweist. Die Peaks sind aufgrund der geringen Kristallitgröße aufgeweitet; unter der Annahme sphärischer Metallpartikel wurde die Halbwertsbreite der Peaks mittels der Scherrergleichung [10] ausgewertet und ergibt 


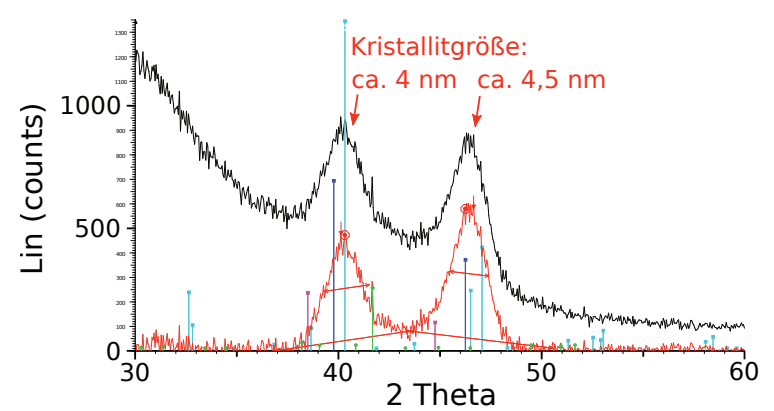

Abbildung 5: XRD-Analyse einer Pt: $\mathrm{Al}_{2} \mathrm{O}_{3}$ Schicht mit Abschätzung der Kristallitgröße anhand der Scherrer-Gleichung.

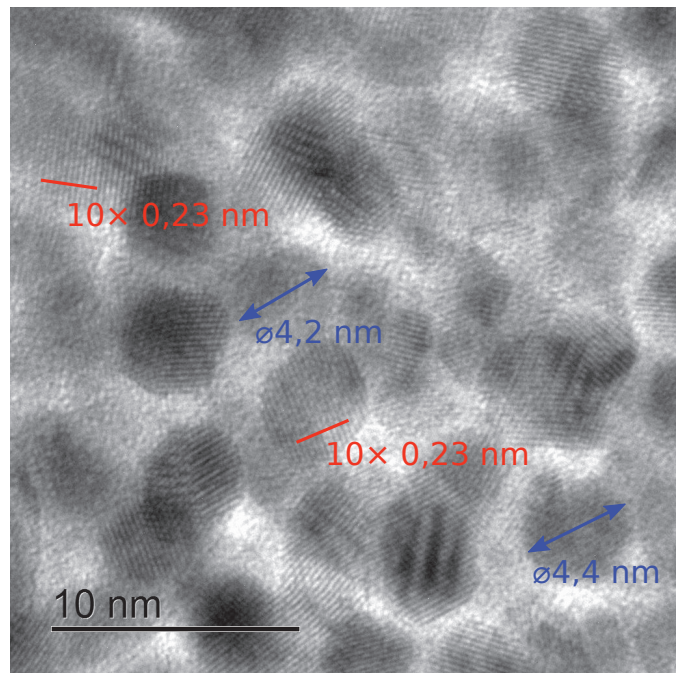

Abbildung 6: TEM-Aufnahme einer dünnen $(\approx$ $20 \mathrm{~nm}) \mathrm{Pt}: \mathrm{Al}_{2} \mathrm{O}_{3}$-Schicht.

für die untersuchten Schichten einen Durchmesser von $(5 \pm 1) \mathrm{nm}$.

In TEM-Untersuchungen wurden sehr dünne Schichten auf einer $\mathrm{Si}_{3} \mathrm{~N}_{4}$-Membran abgeschieden und in Normalrichtung betrachtet. Abbildung 6 zeigt die Aufnahme einer Schicht, die monokristalline, annähernd kreisförmig erscheinende Partikel eines Durchmessers von ca. $4 \mathrm{~nm}$ in einer amorphem Umgebung enthält. Die Auswertung ergibt einen Netzebenenabstand von etwa $0,23 \mathrm{~nm}$, der mit dem erwarteten Netzebenenabstand der (111)-Ebene von Platin übereinstimmt [11]. Da keine Querschnittsproben angefertigt wurden, lässt sich die räumliche Struktur nicht vollständig aufklären; Untersuchungen von Sella [12] und Gibaud [13] legen jedoch nahe, dass für das vorliegende Materialsystem sphärische Partikel entstehen.

\section{Modellbildung}

Aus den vorangegangenen Analysen der Struktur und Ergebnissen aus ähnlichen Prozessen in der Literatur wird abgeleitet, dass in der Schicht sphärische, einkristalline Metallpartikel in einer amorphen Matrix aus $\mathrm{Al}_{2} \mathrm{O}_{3}$ vorliegen.

Für unser Modell wird die geometrische Unordnung in Verbindung mit einem Widerstandsgesetz genutzt, das eine abstandsabhängige Leitfähigkeit zwischen den vorliegenden Metallpartikeln beschreibt. Die Leitfähigkeit zwischen Partikeln setzt sich prinzipiell zusammen aus einer Tunnelleitfähigkeit und einer spezifischen Leitfähigkeit des Trennwandmaterials. Die Leitfähigkeit des Aluminiumoxids ist jedoch mit etwa $10^{12} \Omega \mathrm{m}$ so gering, dass diese vernachlässigt werden kann.

Orientiert an TransmissionselektronenmikroskopAufnahmen der Schicht wird ihre Geometrie abgebildet. Eine rein statistische Gleichverteilung der Partikelkoordinaten liefert kein Bild, das der realen Schicht entspricht, die relativ einheitlich mit Partikeln gefüllt ist. Daher wird für die Modellierung von einer hexagonalen Kugelpackung ausgegangen, bei der jeder Partikel einen festen Platz hat; die Unordnung kann durch Variation (Normalverteilung) der Partikeldurchmesser vorgegeben werden, da sie die Oberflächenabstände der Partikel ändert. Ein Beispiel dieser Struktur ist in Abb. 7 dargestellt. Diese Repräsentation entspricht der Erwartung, dass die räumlich verteilten sphärische Partikel der Schicht in leicht variablem Abstand jeweils etwa 12 nächste Nachbarn besitzen.

Die Anordnung der Partikel bildet mit dem angegebenen Widerstandsgesetz ein Widerstandsnetzwerk. Der Gesamtwiderstand des ungedehnten und des in longitudinaler und transversaler Richtung gedehnten Netzwerks wird berechnet und liefert $k_{L}$ und $k_{T}$. Aufgrund der zufälligen Unordnung variiert das Ergebnis, daher wird die Berechnung als Monte-CarloSimulation für eine Vielzahl zufällig generierter Netzwerke ausgeführt.

Die Elastizitätsmoduln der beiden Materialien werden in der Dehnung berücksichtigt, sodass im Falle von $\mathrm{Pt}_{\mathrm{Al}} \mathrm{Al}_{2} \mathrm{O}_{3}$ das sehr starre Matrixmaterial dafür sorgt, dass die Dehnung zwischen Metallpartikeln geringer ist als die globale Dehnung der Schicht.

Das verwendete Widerstandsgesetz wird der Arbeit von Huth [14] entnommen; seine Ableitung ist im Folgenden zusammengefasst. Es geht von thermisch aktiviertem Transport von Ladungsträgern aus, der durch eine Leitfähigkeit bzw. Widerstand beschrieben wird in der Form

$$
R=R_{0} \exp \left(-\frac{\Delta M}{k_{B} T}\right),
$$

mit der Boltzmannkonstante $k_{B}$, dem Mott Gap $\Delta M$ und der Temperatur $T$. $\Delta M$ lässt sich nähe- 


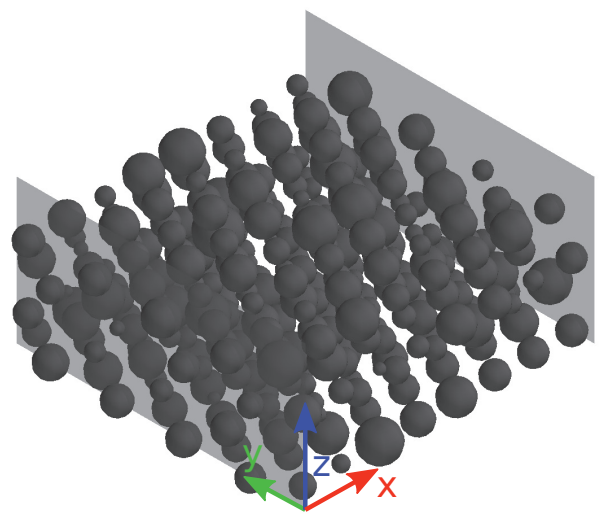

Abbildung 7: Beispielhafte Darstellung einer Anordnung sphärischer Partikel $(n=300)$ variablen Durchmessers für die numerische Berechnung. Die Elektroden sind als Flächen bei $x=0$ und $x=a_{x}$ dargestellt.

rungsweise beschreiben als

$$
\Delta M \approx E_{C} \exp (-\pi g z),
$$

mit der Coulomb-Energie $E_{C}$, die benötigt wird, um ein Elektron aus einem Metallpartikel zu entfernen und in das benachbarte zu transportieren; dem dimensionslosen Coupling $g$ und der Zahl nächster Nachbarn $z$, die für sphärische Partikel in dreidimensionalen Kugelpackung mit 12 angenommen werden kann.

$E_{C}$ kann als Summe der zu überwindenden Austritts- und Eintrittsarbeit verstanden werden und hängt von den Kapazitäten $C_{i}$ und damit den Durchmessern $D_{i}$ der beteiligten Partikel ab:

$$
E_{C}=\frac{e^{2}}{2 C_{0}}+\frac{e^{2}}{2 C_{1}}, \quad \text { mit } C_{i}=2 \pi \epsilon_{0} \epsilon_{r} D_{i}
$$

mit der Elementarladung $e$ und der Permittivität $\epsilon_{0} \epsilon_{r}$. Damit erhält man

$$
E_{C}=e^{2} \frac{D_{0}+D_{1}}{4 \pi \epsilon_{0} \epsilon_{r} D_{0} D_{1}} .
$$

Das Coupling $g$ lässt sich schließlich verknüpfen mit dem Partikelabstand $s$ (Abstand zwischen den Partikeloberflächen):

$$
g=g_{0} \exp (-2 s / \lambda),
$$

mit der Abklingkonstante $\lambda$. Damit lässt sich aus Gleichung (2) ein Widerstandsgesetz finden, das den Tunnelwiderstand und seine Abhängigkeit von den Durchmessern der Partikel und ihrem Abstand zueinander beschreibt.

\section{Simulationsergebnisse und Diskussi- on}

Die Parameter des Widerstandsgesetzes werden angelehnt an [14] gewählt; die Parameter

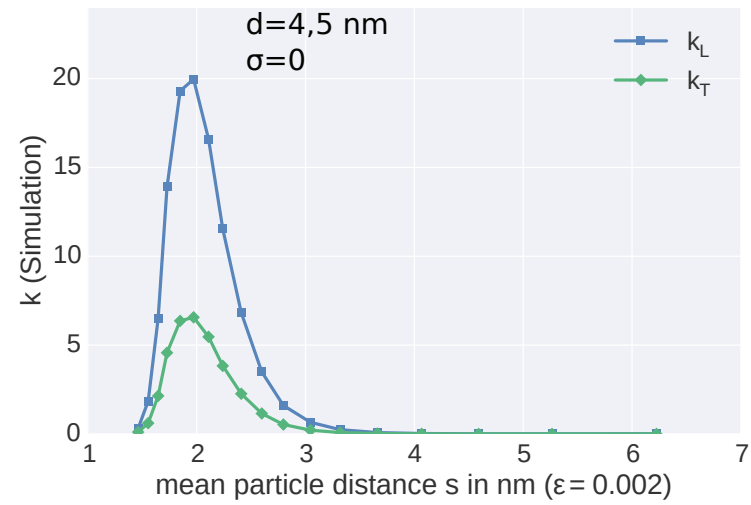

Abbildung 8: Simulationsergebnis zum charakteristischer Verlauf von $k_{L}$ und $k_{T}$ abhängig vom Partikelabstand s.

wurden dort für eine Gold-Nanopartikel-Schicht abgeleitet. Der mittlere Partikeldurchmesser wird an die oben beschriebenen Messergebnisse angepasst: $d=4,5 \mathrm{~nm}$. In der numerischen Rechnung wird typischerweise eine Partikelanzahl von $10^{4}$ verwendet, um Randeffekte durch die begrenzte Größe gering zu halten. In den Plots der Ergebnisse werden die nach Koehler [15] statistisch ermittelten Monte-Carlo-Fehler als 95$\%$-Vertrauensintervalle dargestellt.

Die Unordnung der Partikeldurchmesser wird als ihre Standardabweichung $\sigma$ vorgegeben. Sie entspricht zugleich der Standardabweichung der Partikelabstände und ist ein wesentlicher Parameter für die Simulationsergebnisse. Im Folgenden wird zunächst eine ideale Anordnung der Partikel im hexagonalen Gitter mit $\sigma=0$ gezeigt, anschließend wird der Einfluss eines größeren $\sigma$ demonstriert.

Ideale Ordnung Mit $\sigma=0$ lässt sich die prinzipielle Charakteristik des Widerstandsgesetzes zeigen, da alle ausgewerteten Partikelabstände in der simulierten Schicht identisch sind. In Abb. 8 sind $k_{L}$ und $k_{T}$ abhängig vom mittleren Partikelabstand gezeigt: Für sehr kleine Abstände unter $1,5 \mathrm{~nm}$ gehen die k-Faktoren gegen Null, da das exponentielle Widerstandsgesetz in einem sehr flachen Bereich der Widerstandsänderung liegt. Für sehr große Abstände über $3 \mathrm{~nm}$ geht der k-Faktor ebenfalls gegen Null. Die gleichen Ergebnisse mit dem äquivalenten MetallVolumenanteil als $x$-Achse zeigt Abb. 9: den Verlauf von $k_{L}, k_{T}$ und der Querempfindlichkeit $k_{T} / k_{L}$. Es zeigen sich erhöhte k-Faktoren für einen Bereich zwischen 20 vol- $\%$ bis 40 vol- $\%$ mit einem Maximum von $k_{L}=20$ bei etwa 30 vol- $\%$. Für die gewählten Parameter entspricht der Partikelabstand, der bei 30 vol-\% vorliegt, dem Optimum. Die Querempfindlichkeit liegt durchgehend bei $33 \%$. 


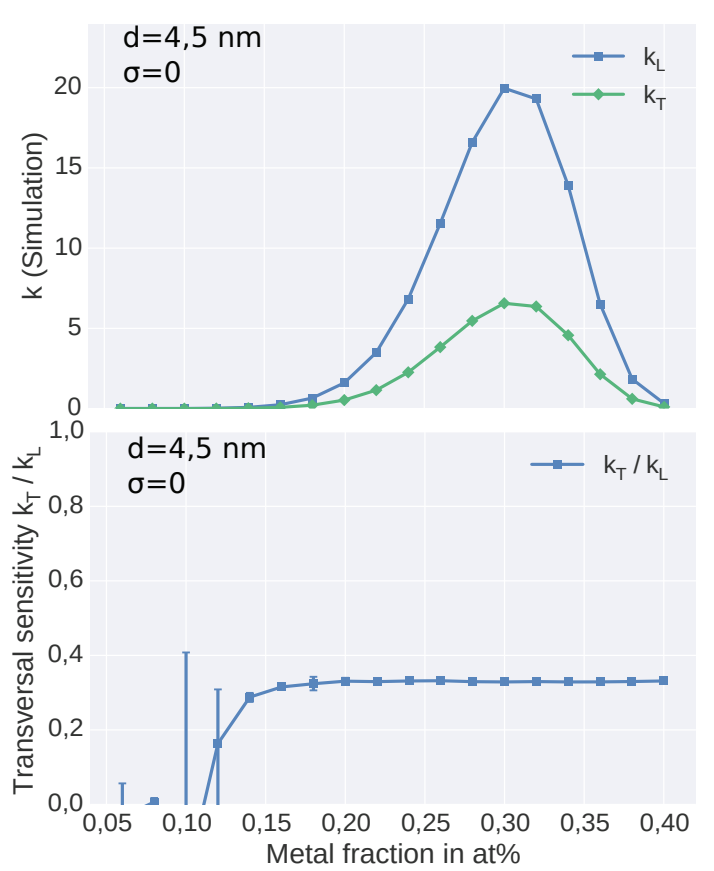

Abbildung 9: Dehnungsempfindlichkeiten $k_{L}$ und $k_{T}$ (oben) und Querempfindlichkeit (unten) in Abhängigkeit vom Metallanteil.

Erhöhte Unordnung Mit einer Standardabweichung von $\sigma=0,5 \mathrm{~nm}$ ändert sich der Verlauf der k-Faktoren, wie in Abb. 10 dargestellt ist. Der Anstieg der k-Faktoren beginnt bereits bei 15 vol$\%$ Metall, da durch die Standardabweichung der Abstände bereits ausreichend kleinere Abstände vorliegen, die einen k-Faktor ermöglichen. Aus dem gleichen Grund steigt die Kurve früher an und erreicht ihr Maximum bei etwa $24 \mathrm{vol}-\%$. Der maximale k-Faktor $k_{L}=13$ ist im Vergleich zur vorigen Kurve reduziert, da durch das größere $\sigma$ nicht alle Abstände den optimalen Wert besitzen, sondern einige größere und einige kleinere vorliegen, die zu einem reduzierten k-Faktor führen. Wie zu erwarten, steigt aufgrund der Unordnung die Querempfindlichkeit der Schicht an, sie liegt für kleinere k-Faktoren bei etwa 0,4 und im Maximum des k-Faktors bei ca. 0,75. Ihr Betrag liegt damit in Übereinstimmung mit den experimentellen Ergebnissen.

Einfluss des Partikeldurchmessers Gegenüber dem vorhergehenden Ergebnis wird nun der mittlere Durchmesser der Partikel verändert. Die (absolute) Standardabweichung bleibt weiterhin bei $\sigma=0,5 \mathrm{~nm}$. Abbildung 11 zeigt Ergebnisse für einen reduzierten Durchmesser von $d=3 \mathrm{~nm}$. Das Maximum des k-Faktors liegt wiederum höher, bei $k_{L}=20$ und ist $\mathrm{zu}$ einem geringeren Metallanteil von etwa 15 vol-\% verschoben.

In den Simulationsrechnungen zu Abb. 12 wur-

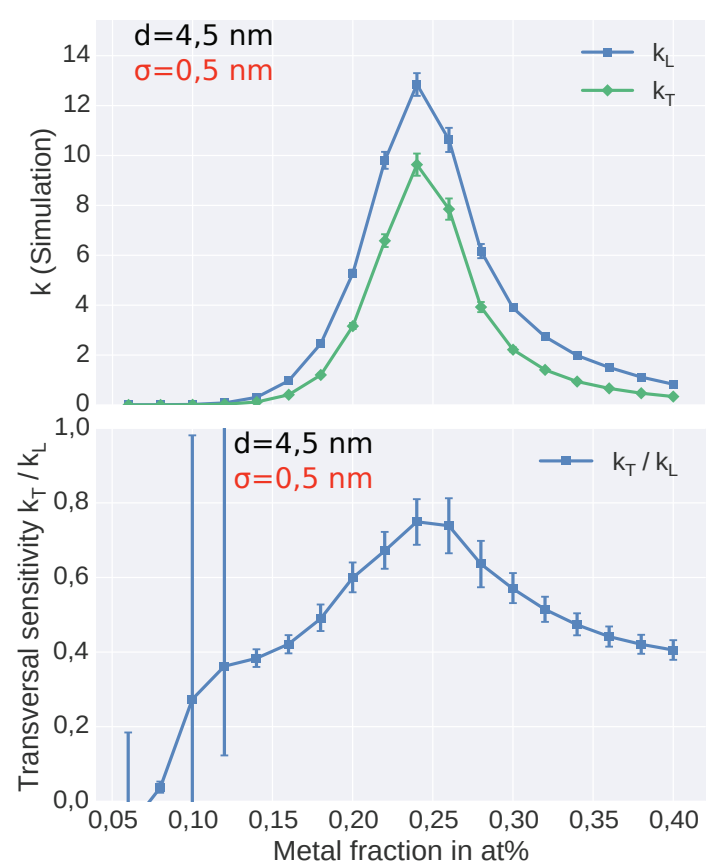

Abbildung 10: Dehnungsempfindlichkeiten $k_{L}$ und $k_{T}$ (oben) und Querempfindlichkeit (unten) in Abhängigkeit vom Metallanteil. Ergebnis für erhöhte Unordnung $\sigma$ der Partikeldurchmesser und-abstände.

de der Durchmesser auf $d=6 \mathrm{~nm}$ erhöht. Das Maximum beträgt nur noch $k_{L} \approx 10$ und liegt bei einem höheren Metallanteil von ca. 31 vol-\%.

Die Querempfindlichkeiten im Bereich der erhöhten k-Faktoren zeigen keine signifikanten Unterschiede für die unterschiedlichen Partikeldurchmesser.

Die Vergleiche der unterschiedlichen Durchmesser zeigen einen starken Einfluss auf Lage und Betrag des maximalen k-Faktors. Dies erschwert den detaillierten Abgleich mit den experimentellen Ergebnissen, liefert allerdings eine mögliche Erklärung für einen breiteren Bereich erhöhter kFaktoren: Bildet die Schicht für kleine Metallanteile kleine Partikel und für größere Metallanteile größere Partikel aus, so führt dies nach der Modellvorstellung dazu, dass über einen weiteren Bereich des Metallanteils ein erhöhter k-Faktor auftritt.

\section{Fazit}

Die granulare Struktur der Cermet-Schicht mit leitfähigen Partikeln und einer isolierenden Matrix führt, wenn die leitfähigen Pfade keine Vorzugsrichtungen besitzen, notwendigerweise zu einer Querempfindlichkeit, da stets ein gewisser Anteil des Elektronentransports in $y$ - und $z$ Richtung, d. h. rechtwinklig zur makroskopischen Stromrichtung ablaufen wird und damit empfind- 


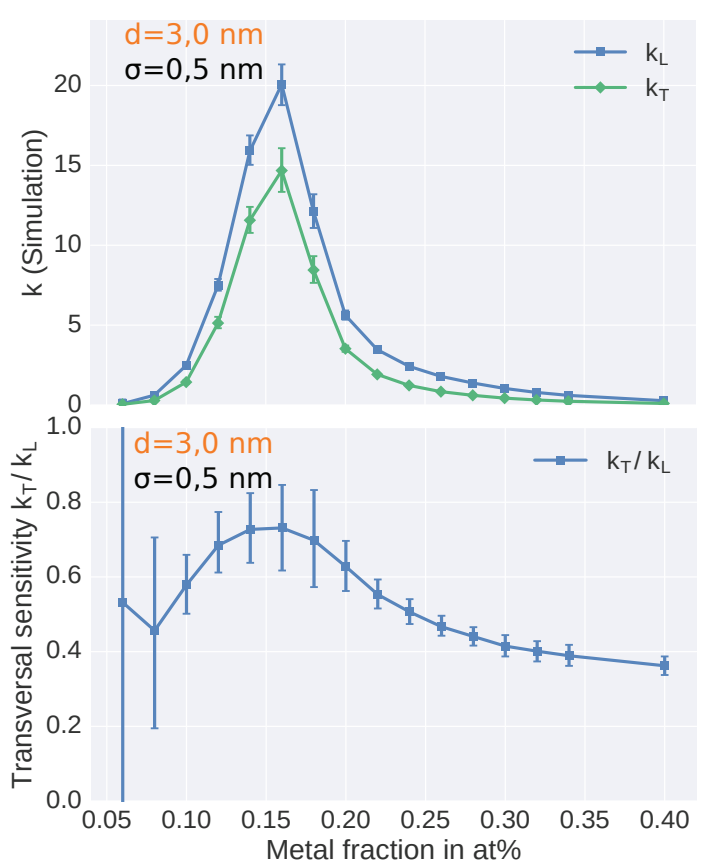

Abbildung 11: Dehnungsempfindlichkeiten $k_{L}$ und $k_{T}$ (oben) und Querempfindlichkeit (unten) in Abhängigkeit vom Metallanteil. Ergebnis für verringerten mittleren Durchmesser, $d=3 \mathrm{~nm}$.

lich ist für Dehnungen in diesen Querrichtungen. Daher ist neben der untersuchten transversalen Empfindlichkeit in $y$-Richtung auch zu erwarten, dass $\mathrm{Pt}_{\mathrm{Al}} \mathrm{Al}_{2} \mathrm{O}_{3}$ bei Deformation in $z$ Richtung, z. B. bei Druck auf die dünne Schicht, eine Widerstandsänderung zeigt.

Diese rein geometrisch bedingten Querempfindlichkeit wird durch zwei weitere, gemeinsam wirkende Einflüsse verstärkt: den Tunnelleitungsmechanismus und die Unordnung in den Partikelabständen. Die Tunnelleitung durch die Schicht enthält eine exponentielle Abhängigkeit des Widerstands vom Partikelabstand. Durch eine Variation der Abstände werden einzelne vergrößert, andere verkleinert. Dies führt in jedem Fall dazu, dass Umwege im Elektronentransport begünstigt werden; durch die exponentielle Widerstandssteigerung bei vergrößerten Abständen wird dies noch verstärkt. In den Simulationsergebnissen wird dieses Phänomen daran sichtbar, dass das Maximum der Querempfindlichkeit mit dem Maximum von $k_{L}$ zusammenfällt - für geringere $k_{L}$ ist auch die Querempfindlichkeit reduziert. Damit trägt der TunnelMechanismus, der den erhöhten k-Faktor bedingt, zugleich zu einer erhöhten Querempfindlichkeit bei.

Für die Erzeugung einer Schicht erscheint es damit geboten, einen Metallanteil zu wählen, der möglichst dem Maximum des k-Faktors entspricht. Durch eine Abweichung davon lässt sich

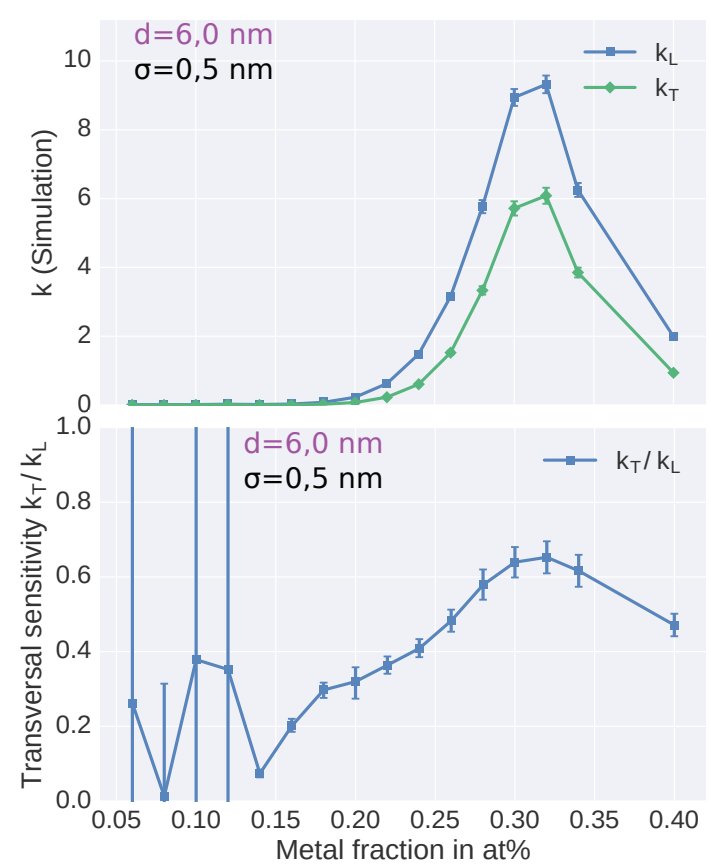

Abbildung 12: Dehnungsempfindlichkeiten $k_{L}$ und $k_{T}$ (oben) und Querempfindlichkeit (unten) in Abhängigkeit vom Metallanteil. Ergebnis für gesteigerten mittleren Durchmesser, $d=6 \mathrm{~nm}$.

zwar bei niedrigerem k-Faktor die Querempfindlichkeit verringern, jedoch nicht ausschalten. Die Querempfindlichkeit hat je nach Dehnungsfall unterschiedliche Auswirkungen: In geeigneten Situationen - beispielsweise einer rein einachsig Dehnung oder einer zweiachsigen Dehnung mit gleichen Vorzeichen - kann die Cermet-Schicht vorteilhaft eingesetzt werden.

\section{Literatur}

[1] G. R. Witt. The electromechanical properties of thin films and the thin film strain gauge. Thin Solid Films, 22(2):133-156, June 1974.

[2] K. Rajanna and M. M. Nayak. Strain Sensors. In Wiley Encyclopedia of Electrical and Electronics Engineering. John Wiley \& Sons, Inc., 2001.

[3] R. Koppert, D. Goettel, O. Freitag-Weber, and G. Schultes. Nickel containing diamond like carbon thin films. Solid State Sciences, 11(10):1797-1800, Oct. 2009.

[4] M. Petersen, U. Heckmann, R. Bandorf, V. Gwozdz, S. Schnabel, G. Bräuer, and C.-P. Klages. Me-DLC films as material for highly sensitive temperature compensated strain gauges. Diamond and Related Materials, 20(5-6):814-818, May 2011.

[5] G. Schultes. Hochempfindliche Dünnschichten für die Druck- und Kraftsensorik auf dem Weg zur technologischen Reife. Sensor Magazin, (2/2015):30-32, May 2015.

[6] A. Tibrewala, E. Peiner, R. Bandorf, S. Biehl, and H. Lüthje. Longitudinal and transversal piezoresistive effect in hydrogenated amorphous carbon 
films. Thin Solid Films, 515(20-21):8028-8033, July 2007.

[7] C. Grimaldi, P. Ryser, and S. Strässler. Anisotropic random resistor networks: A model for piezoresistive response of thick-film resistors. Journal of Applied Physics, 92(4):1981-1986, Aug. 2002.

[8] C. Grimaldi, P. Ryser, and S. Strässler. Longitudinal and transverse piezoresistive response of granular metals. Physical Review B, 64(6):064201, July 2001.

[9] C.-W. Jiang, I.-C. Ni, S.-D. Tzeng, and W. Kuo. Nearly isotropic piezoresistive response due to charge detour conduction in nanoparticle thin films. Scientific Reports, 5:11939, July 2015.

[10] A. L. Patterson. The Scherrer Formula for X-Ray Particle Size Determination. Physical Review, 56(10):978-982, Nov. 1939.

[11] C. Solliard and M. Flueli. Surface stress and size effect on the lattice parameter in small particles of gold and platinum. Surface Science, 156, Part 1:487-494, June 1985.

[12] C. Sella, M. Mâaza, B. Pardo, F. Dunsteter, J. C. Martin, M. C. Sainte Catherine, and A. Kaba. Structural investigation of Pt-Al2o3 co-sputtered nano-cermet films studied by small angle $X$ ray scattering, grazing angle $X$-ray reflectometry, TEM and AFM. Surface and Coatings Technology, 97(1-3):603-610, Dec. 1997.

[13] A. Gibaud, S. Hazra, C. Sella, P. Laffez, A. Désert, A. Naudon, and G. Van Tendeloo. Particle layering in the ceramic-metal thin film PtAl2o3. Physical Review B, 63(19):193407, Apr. 2001.

[14] M. Huth. Granular metals: From electronic correlations to strain-sensing applications. Journal of Applied Physics, 107(11):113709, June 2010.

[15] E. Koehler, E. Brown, and S. J.-P. A. Haneuse. On the Assessment of Monte Carlo Error in Simulation-Based Statistical Analyses. The American Statistician, 63(2):155-162, May 2009. 